\title{
Les interdits religieux dans les rations militaires
}

Rations halal et casher dans les armées française et américaine

Religious prohibitions in military rations: halal and kosher rations in the French and American armies

\section{Vincent Moriniaux}

\section{(2) OpenEdition}

1 Journals

\section{Édition électronique}

URL : http://journals.openedition.org/artefact/2849

DOI : 10.4000/artefact.2849

ISSN : 2606-9245

Éditeur:

Association Artefact. Techniques histoire et sciences humaines, Presses universitaires du Midi

\section{Édition imprimée}

Date de publication : 15 mars 2019

Pagination : 79-104

ISBN : 978-2-8107-0623-5

ISSN : 2273-0753

\section{Référence électronique}

Vincent Moriniaux, «Les interdits religieux dans les rations militaires », Artefact [En ligne], 9 | 2018, mis en ligne le 04 mars 2020, consulté le 27 novembre 2020. URL : http://journals.openedition.org/ artefact/2849; DOI : https://doi.org/10.4000/artefact.2849

\section{(c) $(1)$}

Artefact, Techniques, histoire et sciences humaines est mise à disposition selon les termes de la Licence Creative Commons Attribution - Pas d'Utilisation Commerciale - Pas de Modification 4.0 International. 


\title{
Les interdits religieux dans les rations militaires
}

\author{
Rations halal et casher dans les armées française \\ et américaine
}

Vincent Moriniaux

\section{Résumé}

En France, où la conception de la laïcité est assez rigide, la question de l'alimentation des soldats musulmans et juifs pratiquants est essentielle mais objet de polémiques. Cette recherche, fondée sur les documents officiels accessibles et sur des entretiens avec les acteurs, tente de comprendre comment se mêlent les évolutions concomitantes de la place des musulmans dans l'armée depuis la Première Guerre mondiale, des techniques de ravitaillement et, enfin, de la pratique et de la perception de la religion musulmane pour aboutir au surgissement en 2010 des premières rations halal, bien après les premières rations casher. Afin de mieux percevoir ce qui se joue dans l'armée française, est enfin menée une courte comparaison avec l'armée des États-Unis d'Amérique.

\section{Mots-clés}

ration, armée française, laïcité, islam, judaïsme

99 Vincent Moriniaux, « Les interdits religieux dans les rations militaires : rations halal et casher dans les armées française et américaine », Artefact, 9, 2018, p. 79-104. 


\section{Religious prohibitions in military. rations: halal and kosher rations in the French and American armies}

\section{Abstract}

In France, where conception of secularism is quite rigidly defined, feeding the Muslim or Jewish troops is a key issue but also subject of controversial discussions. This research, based on publicly available official documents and interviews with actors, aims at understanding how several simultaneous developments interact to explain why halal rations have been existing only since 2010 onwards while kosher rations have been available for a much longer time. The answer involved the increasing number and role of Muslims in the army since the First World War, the developments in food catering, the evolving religious practices as well as the perception of Muslim faith. In order to identify what is at stake in the French Army, a short comparison will be made with the situation in the US Army. 


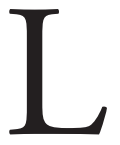
a question des interdits religieux dans les rations militaires pourrait paraître une question bien secondaire. Bien au contraire ! En ces temps de crispation identitaire et de pertes des repères religieux traditionnels ${ }^{1}$, cette question, pourtant déjà ancienne, trouve un nouvel écho. Toutefois, force est de constater que les récents ouvrages de spécialistes de la laïcité parlent davantage de l'école et des cantines scolaires que de l'armée et de la ration des soldats ${ }^{2}$. La République française déclare respecter les croyances de ses soldats ${ }^{3}$ de même que leur appartenance ethnique, c'est la moindre des considérations à l'égard d'un militaire appelé à mourir pour son pays. Pourtant, dans les 39 pages du livret Expliquer la laïcité française : une pédagogie par l'exemple de la "laïcité militaire ", édité en novembre 2017 par la DGRIS du ministère des Armées ${ }^{4}$, la question du respect des prescriptions alimentaires religieuses est traitée seulement en une ligne et une note :

Les militaires ont la possibilité, s'ils le souhaitent, de se conformer à des prescriptions religieuses d'ordre alimentaire et peuvent bénéficier de rations sans porc voire, dans la mesure du possible, certifiées halal ou casher (circulaire Pierre Joxe').

1. Voir Olivier Roy, La Sainte Ignorance, Paris, Seuil, 2012. Selon l'auteur, la perte des repères viendrait d'une disjonction croissante entre religion et culture, sous l'effet combiné de la mondialisation (métissages culturels) et des migrations (déracinement des peuples de leur culture d'origine). Le livre porte comme sous-titre : "Le temps de la religion sans culture ».

2. Il n'y a par exemple aucun chapitre sur l'armée dans La Laïcité, une valeur d'aujourd'hui ? Contestations et renégociations du modèle français, ouvrage dirigé par Jean Baudouin et Philippe Portier en 2001 aux Presses universitaires de Rennes (coll. Res publica). De même, l'historien et sociologue Émile Poulat, spécialiste français de la question, ne consacre-t-il pas un seul chapitre à la laïcité militaire dans les 416 pages de son livre La France est une république lä̈que, Paris, Berg international, coll. Faits et représentation, 2003.

3. L'article 2 de la loi de 1905 qui porte séparation des Églises et de l'État concerne des dispositions particulières pour les personnes détenues dans les prisons, pour les hôpitaux et les internats scolaires publics - tous les lieux où un individu ne peut accéder librement aux lieux de culte traditionnels. Dans ces cas, l'État doit donner un accès à un aumônier désigné par ses soins. La loi de 1905 fait pendant à une loi semblable de 1880 qui donne ces mêmes droits aux membres de l'armée française. Il existe actuellement 38 aumôniers musulmans dans l'armée, aux côtés des 219 aumôniers catholiques, 26 protestants et 9 israélites (sans compter les aumôniers des différentes religions à statut civil).

4. Direction générale des relations internationales. Le livret est téléchargeable à l'adresse : https:// www.defense.gouv.fr/espanol/dgris/presentation/evenements/expliquer-la-laicite-francaise.

5. La circulaire Pierre Joxe institutionnalise une pratique séculaire de proposer aux soldats des rations sans porc. 
La mention est pour le moins lapidaire. Il paraît donc intéressant de tenter une analyse de cette pratique présentée comme " séculaire " de l'armée française qui, tout en semblant couler de source, a connu des évolutions récentes. Parmi ces dernières, la plus importante est l'introduction en 2010 de rations de combat individuelles réchauffables (RCIR) halal. Deux citations témoignent de ce que la perception des soldats eux-mêmes à ce sujet est floue : "Avant 2005 et la création de l'aumônerie militaire musulmane, il y avait du casher mais pas de halal dans l'armée française ${ }^{6}$." "Fils d'un ancien de la Coloniale, j'ai toujours connu les rations destinées aux troupes de confession musulmane, et cela n'avait rien de choquant à l'époque $^{7}$ ». Ces deux citations posent le problème : comment expliquer que les mesures anciennes ne soient plus perçues comme suffisantes ou efficaces aujourd'hui ? Qu'est-ce qui a changé ? Est-ce un problème technique ou religieux ? Les deux ? Pourquoi cette différence entre le casher et le halal ? Comment cela se passe-t-il à l'étranger, notamment aux ÉtatsUnis ? L'exemple des États-Unis est ici essentiel parce que s'avère connue l'influence de nos alliés lors des deux dernières guerres, tout spécialement la Seconde, sur l'évolution de nos conceptions en matière de ravitaillement.

\section{Une recherche sans statistiques?}

Il convient tout d'abord de poser quelques remarques méthodologiques. La première concerne le nombre. Afin de savoir si la question des rations religieuses est secondaire ou non, il faudrait pouvoir disposer de statistiques. En France, la loi interdisant toute statistique fondée sur l'appartenance à une religion ${ }^{8}$, il est évidemment impossible de dépasser le stade des estimations.

6. Interview de Nadir Medhi (aumônier militaire musulman en région parisienne) par Vincent Moriniaux, octobre 2017.

7. Commentaire à l'article "Larzul va fournir à l'Éloca des plats cuisinés appertisés halal en barquettes de $300 \mathrm{~g}$ » du 27 avril 2017 sur le blog de Philippe Chapleau, Lignes de Défense : http:// lignesdedefense.blogs.ouest-france.fr/archive/2017/04/25/fourniture-de-plats-cuisines-appertises-halal-en-barquettes-17986.html, consulté en novembre 2017.

8. Non pas la loi de 1905 mais la loi informatique et liberté de 1978. La loi française interdit depuis 1872 de questionner quelqu'un sur sa religion lors des recensements effectués par les organismes publics. La République provisoire estimait en effet qu'il s'agissait d'une question privée. Un principe réaffirmé dans la loi du 6 janvier 1978 (article 8) : «Il est interdit de collecter ou de traiter des données à caractère personnel qui font apparaître, directement ou indirectement, les origines raciales ou ethniques, les opinions politiques, philosophiques ou religieuses... ». 
Pour les soldats de confession juive, si les études sur leur rôle dans l'armée française en 1914-1918 existent ${ }^{9}$, il n'y a en revanche aucune estimation de leur effectif actuel. Le blog de l'aumônerie israélite des armées ${ }^{10}$, actif de 2009 à 2014, s'avère muet sur le sujet.

Quant au nombre de musulmans, il est relativement bien évalué pour la période des deux guerres mondiales ${ }^{11}$, mais il n'y a pas davantage de chiffres officiels aujourd'hui. Une estimation d'un spécialiste de l'islam, Olivier Roy, dans une interview au Monde des Religions en 2015, circule $^{12}: 10 \%$, soit quelque 20000 soldats sur un effectif total des armées de 205121 personnes en $2017^{13}$. Selon le sociologue, la présence de musulmans est plus importante aujourd'hui, ne serait-ce que parce que l'armée est, pour les enfants issus de l'immigration, un gage d'intégration dans la société et un avenir professionnel pour des jeunes n'ayant pas toujours brillé dans le système scolaire. La présence de musulmans est surtout plus diffuse. Il n'existe plus de régiments de Marocains ou de tirailleurs sénégalais maintenus en un groupe ethnique homogène. Les recrues de foi musulmane sont aujourd'hui mieux réparties, même si les rapports qui se succèdent soulignent toujours leur position souvent inférieure, notamment en raison de leur bagage initial (rapport Biville en 1990 et rapport Aït Hocine

9. Par exemple, les ouvrages de l'historien Philippe-E. LANDAu, comme Les Juifs de France et la Grande Guerre, un patriotisme républicain, Paris, CNRS Éditions, 1999. Philippe Landau évalue à 36000 le nombre de juifs mobilisés en 1914-1918 côté français, sur 180000 personnes juives en France et en Algérie (côté allemand : plus de 96 000, sur une population juive allemande totale de 480000 personnes).

10. http://aumonerieisraelitedesarmees.blogspot.com

11. En 1914-1918, l'armée française regroupe dans ses rangs environ 300000 combattants originaires du Maghreb, auxquels il faut ajouter des milliers de soldats musulmans parmi les quelques 180000 tirailleurs venus d'Afrique noire. Michel RenArD, " Le religieux musulman et l'armée française (1914-1920) ", Études coloniales, août 2014, revue en ligne : http://etudescoloniales.canalblog.com/archives/2014/08/23/30279901.html. En 1939, les "indigènes d'Afrique du Nord " comptent 127875 engagés et appelés d'Algérie (53\%), du Maroc (28\%) et de Tunisie (19\%). En décembre 2014, François Hollande a inauguré à la Grande mosquée de Paris un mémorial destiné aux 100000 soldats musulmans morts pour la France lors des deux guerres mondiales.

12. "Nombreux sont les musulmans qui désormais appartiennent aux administrations publiques. Sur le porte-avion Charles de Gaulle, par exemple, on compte 300 musulmans sur un équipage de 3000. C'est une proportion que l'on retrouve dans le reste de l'armée. Il suffit de compter le nombre d'aumôniers musulmans pour s'en rendre compte. " Olivier Roy, "La laïcité est devenue une idéologie ", Le Monde des Religions, 13 mars 2015, consulté sur http://www.lemondedesreligions.fr/ actualite/olivier-roy-la-laicite-est-devenue-une-ideologie-13-03-2015-4600_118.php.

13. Données extraites de "Chiffres clés de la Défense - 2017 " : https://www.defense.gouv.fr/ actualites/articles/chiffres-cles-de-la-defense-2017. 
en 2005). Il ne faut pas occulter non plus les convertis qui ne sont pas du tout identifiables par leur patronyme et ne figurent pas parmi les moins revendicatifs sur la question du halal.

La deuxième remarque découle directement de la première : il est très difficile d'enquêter sur cette question car qui dit rations dit possibilité de compter les boîtes et donc d'alimenter les estimations, non dénuées d'arrière-pensées ${ }^{14}$. Pourtant, des acteurs, religieux comme l'imam Nadir Mehdi et le rabbin Moïse Taïeb, ou civils (cadres de l'ÉLOCA et du CESCOF, voir plus loin), ont accepté de répondre à nos questions. La présente étude se fonde également sur l'exploration de l'Internet au sujet des marchés publics ${ }^{15}$, sur les blogs de soldats ou d'anciens soldats ${ }^{16}$ à la recherche d'avis et de témoignages. Dans la littérature scientifique, rien ne transparait sur le sujet spécifique de l'alimentation confessionnelle dans les rangs militaires, en revanche existent des études soit sur l'alimentation du soldat, dans une perspective historique ${ }^{17}$, soit sur la foi du soldat, dans une perspective sociologique ${ }^{18}$ et en rapport avec son intégration quand il est issu de l'immigration ${ }^{19}$.

Enfin, parmi les remarques préliminaires, il convient de rappeler ce qui unit et ce qui oppose les interdits de l'islam et du judaïsme. Il n'y a en pratique que deux seuls vrais points communs : l'interdit de la viande de porc et l'obligation de l'abattage rituel. La kashrout (code alimentaire juif) est à la fois beaucoup plus précise, beaucoup plus contraignante (elle concerne

14. Le nombre de musulmans dans une unité pourrait être facilement connu grâce à l'"État des rationnaires ", document mis à jour quotidiennement sur lequel sont précisés le nombre de convives et les régimes particuliers de façon non nominative. Ce document est édité par le secrétariat de l'unité et signé par le commandant d'unité. Toutefois, ce document demeure inaccessible au chercheur.

15. Notamment les sites https://www.marches-publics.gouv.fr et https://centraledesmarches.com.

16. Notamment les sites http://www.forum-militaire.fr et http://lignesdedefense.blogs.ouestfrance.fr.

17. Emmanuelle Cronier, "Feeding Muslim troops during the First World War ", in Xavier Bougarel, Raphaëlle Branche et Cloé Drieu (dir.), Far from Jihad: Combatants of Muslim Origin in European Armies in the 20th Century, Londres, Bloomsbury Academic, 2017, p. 48-70. Pierre Eveno, "L'alimentation du soldat pendant la Première Guerre mondiale ", Carnet de la sabretache, $\mathrm{n}^{\circ} 210$, mars 2017, p. 11-17.

18. Catherine Wintol de Wenden, "L'islam dans l'armée ", Cahiers de la Méditerranée, n 76, 2008, p. 65-88.

19. Elyamine Settoul, "Musulmans dans les armées françaises, Entre banalisation institutionnelle et altérité imaginaire ", Centre d'information et d'études sur les migrations internationales, Migrations Société, 2008/6, n 120. 
non seulement le produit consommé et la mise à mort de l'animal mais aussi les ustensiles de cuisine et la succession des mets : séparation laitviande) et beaucoup mieux organisée (par le Consistoire israélite de France fondé en 1808 par Napoléon ${ }^{\text {Ir) }}$ ) que les règles suivies par les populations musulmanes. Ceci se voit bien aujourd'hui avec la multiplication des certifications halal et l'absence d'une autorité religieuse claire sur le sujet ${ }^{20}$. Selon le Coran (sourate 5, 5), la nourriture des Gens du Livre est halal. Ce qui est casher est halal (mais pas l'inverse). La viande issue des abattoirs en terre chrétienne, sauf la viande de porc, ne posait pas de problème pour un musulman avant la généralisation de l'étourdissement préalable dans les abattoirs français en $1964^{21}$. Enfin, dans les deux religions, Dieu pardonne en cas d'absolue nécessité.

Une dernière remarque découle sans doute de la précédente. La pratique est aussi très différente entre les juifs et les musulmans : 10 à $15 \%$ des juifs en France consommeraient strictement casher, ce qui se révèle très compliqué, alors que neuf personnes originaires du Maghreb sur dix achèteraient du halal $^{22}$, dont les produits sont présents dans tous les supermarchés.

\section{De la roulante à la barquette : évolution des méthodes de ravitaillement}

L'évolution de la question du respect des prescriptions religieuses en matière alimentaire, découle d'abord de l'évolution des méthodes de ravitaillement.

\section{La roulante en 1914-1918}

L'alimentation du soldat était en 1914-1918 largement mise en œuvre collectivement. Apparue en 1915, la roulante (poétiquement dénommée par les poilus la bitumeuse, le canon à rata, la mitrailleuse à haricots, etc.) en est le symbole ${ }^{23}$. Les conserves en boîtes métalliques ne se répandent

20. Florence Bergeaud-Blackler, Le Marché halal ou l'invention d'une tradition, Paris, Seuil, 2017. 21. Le décret du 16 avril 1964, pris après la campagne menée par Jacqueline Gilardoni (fondatrice en 1961 de l'Euvre d'assistance aux bêtes d'abattoirs), le rend obligatoire.

22. Les études sur les habitudes de consommation des deux communautés ne sont jamais strictement comparables et ces estimations ne peuvent être, au mieux, que des ordres de grandeur.

23. Au départ, la cuisine mobile qu'est la roulante n'est pas desservie par un véritable cuisinier. Il faut en effet attendre un décret du 29 mai 1936 pour voir la création d'un personnel spécialiste de 
vraiment ${ }^{24}$ qu'après la guerre de 1870 , sans que cela ne devienne pour autant vraiment la règle. Avant 1900, l'administration militaire achète des conserves à l'étranger, notamment en Australie et aux États-Unis. Ce sont des conserves collectives, viande conditionnée en boîte d'un ou deux kilogrammes, à partager.

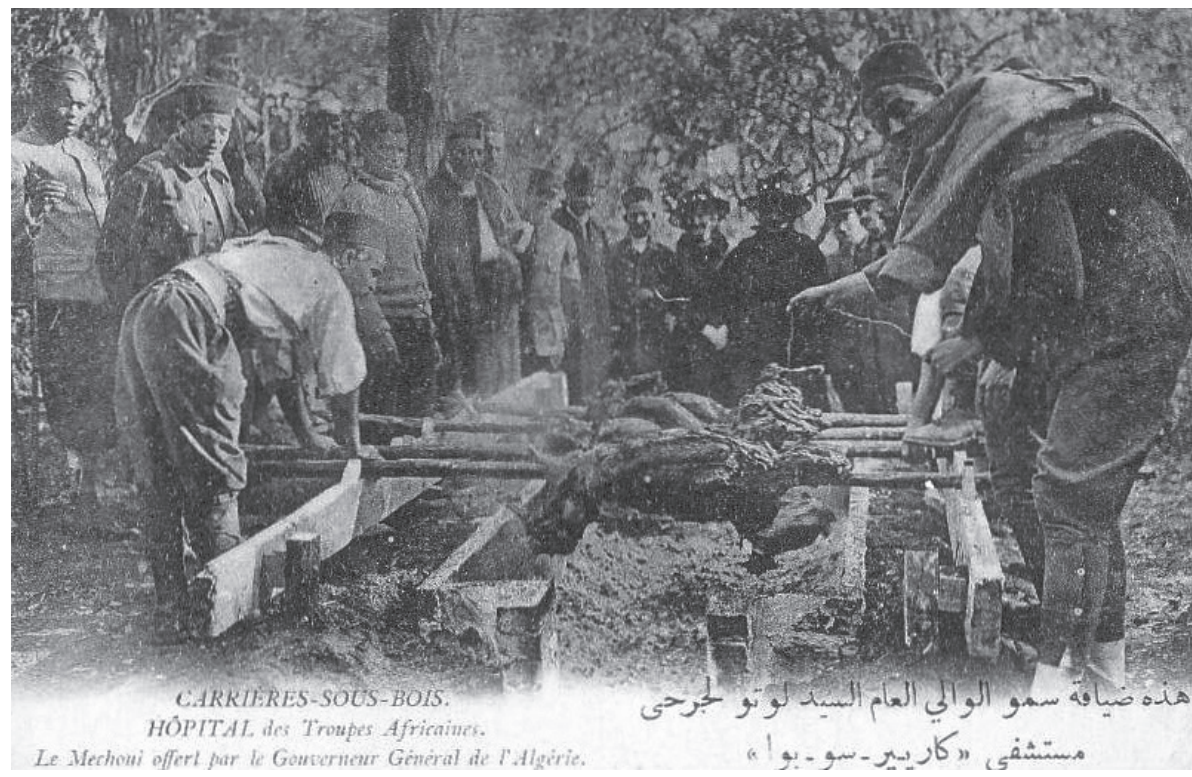

Fig. 1. - La cuisson des moutons à l'hôpital militaire musulman de Carrières-sous-Bois (Seine-et-Oise, aujourd'hui Yvelines), en novembre 1917

Méchoui offert par le gouverneur général de l’Algérie. Carte postale d’époque.

La Contemporaine : http://www.lacontemporaine.fr/

Les adaptations pour les soldats musulmans consistent donc seulement dans la fourniture de la matière première alimentaire. Par exemple, sont donnés du thé vert et de la menthe séchée en remplacement du vin aux troupes coloniales d'Afrique du Nord et des noix de Kola aux tirailleurs

cuisiniers militaires, titulaire d'un brevet délivré à l'issue d'un enseignement, théorique et pratique, et d'un examen.

24. À partir de 1806, la marine impériale de Napoléon commande certes des conserves à Nicolas Appert mais celles-ci sont dans des bouteilles de verre type champagne (Nicolas Appert était Champenois...) puis, ultérieurement, dans des bocaux de verre. Malgré l'évident risque de casse que représente le verre, Appert reste réticent à utiliser des boîtes en fer-blanc. 
sénégalais. Il est fourni une viande sur pied autre que le porc ${ }^{25}$. Les cartes postales anciennes, qui ont popularisé cette image des troupes coloniales autour d'un mouton sacrifié (Fig. 1), sont un matériel utile pour le chercheur $^{26}$. Un court film dans les archives allemandes, sans doute tourné pendant une fête de l'Aïd-el-Fitr, dans les camps de prisonniers pour les troupes indigènes françaises et anglaises à Zossen (Allemagne) montre la mise à mort rituelle de moutons devant une assemblée de soldats français musulmans ${ }^{27}$.

Dès les premières semaines du conflit en 1914, est lancée la production de conserves de viande avec du riz dans 17 usines autour de Paris ${ }^{28}$. L'État réquisitionne en 1915 les usines $\mathrm{Kub}^{29}$ (sises quai de la Loire à Paris) pour la fabrication de potages. En 1916, s'effectue l'achat de conserves de bœuf en Argentine puis, en 1917, de conserves de saumon aux États-Unis. Cependant, la grande question réside surtout dans la gestion de la viande congelée car demeure toujours privilégié le ravitaillement collectif d'aliments bruts.

Sur le front de l'Est, le ravitaillement pour les troupes musulmanes s'effectue assez bien, à partir notamment d'élevages de moutons localisés dans le sud de la France et envoyés vivants aux soldats. L'intendance fournit un mouton et il suffit d'un musulman, d'un couteau et de la qibla pour que la viande soit halal ${ }^{30}$. Cependant, sur le théâtre des Balkans et du

25. E. Cronier, « Feeding Muslim troops during the First World War », op. cit.; M. Renard, « Le religieux musulman et l'armée française (1914-1920) », op. cit.

26. Voir E. Cronier, «Feeding Muslim troops during the First World War », op. cit. et id., «Les particularismes culturels, support du moral des troupes alliées pendant la Première Guerre mondiale ", in Michaël Bourlet et al., Petites patries dans la Grande Guerre, Rennes, Presses universitaires de Rennes, 2013, p. 227-238.

27. Film Beiramfest im Mohammedaner Gefangenenlager (Halbmond und Weinbergslager) zu Wünsdorf bei Zossen, Bundesarchiv B 133242-1, visible également à l'adresse : https://www.youtube.com/ watch ? $\mathrm{v}=6 \mathrm{nx} V \mathrm{r} 4 \mathrm{qk} l \mathrm{~N} 4$.

28. Jacques Grasser, «Le ravitaillement de la place de Verdun », in Gérard Canini, Les Fronts invisibles : Nourrir - Fournir - Soigner, actes du colloque international sur " La Logistique des Armées au combat pendant la Première Guerre mondiale ", organisé à Verdun les 6, 7 et 8 juin 1980, à l'initiative du Comité national du souvenir de Verdun en liaison avec l'université de Nancy, Nancy, Presses universitaires de Nancy, 1984, p. 349-370.

29. Contrairement aux idées et à la rumeur publique colportées à l'époque, le bouillon Kub, inventé par le Marnais Claude Émile Théodore Urban, est bien un brevet français, déposé en 1907.

30. Pour le casher, il faut un rabbin qui doit effectuer une inspection minutieuse de la carcasse pour rechercher d'éventuelles tares. 
Proche-Orient, le ravitaillement se révèle beaucoup plus délicat et les soldats musulmans reçoivent alors les mêmes conserves que tous les soldats.

Pourtant, rien n'est encore systématisé. Par exemple, il n'existe pas de boîtes de ration, seulement des sachets en toile pour le pain et le sucre. La boîte en fer n'est en usage qu'à la fin de la guerre. La véritable révolution vient de la Deuxième Guerre mondiale et de l'influence des Américains avec leur ration K, du nom du physiologiste Ancel Keys en charge de l'équilibre nutritionnel de l'armée en 1941, bientôt copiée en France avec la ration type 46. Tout change donc avec la ration, en boîte puis en sachet, c'est-àdire lorsque celle-ci devient industrialisée.

\section{3-1945 : quand les Américains nourrissent les Français}

Lors du deuxième conflit mondial, les Forces françaises libres à Londres sont prises en charge par les Britanniques. À partir de l'armistice de juin 1940, les troupes d'Afrique du Nord et d'Outre-mer ne sont plus ravitaillées par la métropole. Quand les Américains débarquent le 8 novembre 1942 en Afrique du Nord, ils apportent leur système d'alimentation du soldat en campagne. Le Corps expéditionnaire français créé à Alger en 1943 est intégré à la $5^{\mathrm{e}}$ Armée américaine. Ce sont donc les Américains qui adaptent leur ration à la présence d'une forte population musulmane dans l'armée française. Ils créent la French moslem ration, tandis qu'ils adaptent aussi leur ration $\mathrm{K}$ pour les Français : davantage de pain et de vin mais, pour compenser, moins de beurre, de lait et d'œufs. Les soldats musulmans se plaignent de la monotonie de leurs rations et le commandement français est obligé de créer une prime d'alimentation en francs, distribuée à chaque homme pour qu'il puisse acheter localement des fruits et des légumes ${ }^{31}$. Le cours de la guerre s'accompagne ainsi d'une évolution des techniques de ravitaillement. Schématiquement, s'effectue le passage de la tranchée à la guerre mobile et donc de la roulante à la barquette individuelle qui peut bien mieux répondre aux besoins de combattants, dispersés sur le terrain, qui doivent transporter leur alimentation dans un volume limité et un poids réduit au minimum.

31. Claire Мıот, "Une police des âmes ? L'islam dans l'armée française en 1944-1945 ", Revue historique des armées, 4/2017, n² 289, p. 40-54. 


\section{De 1945 à nos jours : toujours des barquettes mais de plus en plus de choix}

À l'époque du deuxième conflit mondial, il existe trois menus : un "bœuf", un " porc" et un "poisson ». Soit deux menus consommables indifféremment par les militaires musulmans et juifs, à condition de n'être regardant ni sur la rigueur de la kashrout, ni sur l'abattage rituel.

En 1951, il existe désormais une palette de 10 menus qui passent à 12 en 1966, avec 8 menus type E (pour Européens) et 4 menus type M (pour musulmans) cette année-là. En 1986, est servie la ration de combat individuelle réchauffable $(\mathrm{RCIR})^{32}$, avec un petit réchaud et son combustible, pour les 10 menus dont 5 ne contiennent pas de porc. Le total s'élève à 14 menus en 2002 puis à 17 en 2017.

\section{Vers une « banalisation du fait religieux »? Évolution des perceptions}

Au cours des conflits mondiaux, l'État-Major a, autant que possible, respecté les prescriptions alimentaires et, plus largement, les pratiques religieuses de tous ses combattants pour une raison principale : il s'agissait, en ce qui concerne les musulmans, de groupes ethniques issus des colonies, constitués pour l'essentiel d'appelés et qu'il convenait d'intégrer dans un même collectif, sur la base de l'égalité, afin de s'assurer le soutien de l'empire colonial à l'effort de guerre. C'était relativement aisé sur les fronts européens. Aujourd'hui, les soldats musulmans sont des engagés volontaires, citoyens français à part entière quand ils s'engagent (à l'exception de la Légion étrangère) et ils sont projetés en OPEX (opérations extérieures) sur des terrains lointains et souvent en terre d'islam. Il ne s'agit donc plus, pour l'État-major, de gérer un groupe relativement homogène mais de permettre à l'individu de servir son pays tout en conservant le libre exercice de sa religion.

32. La RCIR est la ration standard pour une consommation quotidienne d'un combattant. D'un poids de 1,75 kilogramme, elle se conserve deux ans maximum. Sa valeur énergétique est d'environ $3500 \mathrm{kcal} / 14644 \mathrm{~kJ}$ (protides, $13 \%$; lipides, $32 \%$; glucides, $55 \%$ ). 


\section{Grande Guerre : s'assurer le soutien des colonisés}

Ladaptation aux prescriptions religieuses semblait au début du $\mathrm{xx}^{\mathrm{e}}$ siècle une pratique aisée. La perception des troupes coloniales est d'ailleurs généralement bonne, pour autant que l'historien puisse en juger par les rapports des gradés et les lettres des soldats ${ }^{33}$. Il est vrai que les soldats issus des colonies placent souvent la question de la quantité avant celle du respect des interdits. Les tensions résultent le plus souvent de l'absence de substitut au gras de porc, lorsque l'approvisionnement en huile de palme ou d'arachide n'est pas possible ou des ruptures dans l'approvisionnement en produits traditionnels comme la graine de Kola.

Quant à la pratique du jeûne, elle ne semble pas avoir posé de gros problèmes. Il y a un siècle pourtant, le ramadan se produisit durant l'été. En effet, en 1915, le mois sacré de l'islam s'est déroulé du 13 juillet au 12 août. Ceci conduisit, en pleine Grande Guerre, l'armée française à s'adapter à la pratique religieuse de ses soldats musulmans. Dès le 26 juin 1915, le ministère de la Guerre prend des dispositions à l'approche du ramadan ${ }^{34}$ :

À partir du 12 juillet au soir, les militaires musulmans qui auront déclaré vouloir jeûner pourront prendre leurs repas aux heures suivantes : café du matin reporté au coucher du soleil. Déjeuner trente minutes environ après le café. Dîner vers 23 heures. En pays musulman, la rupture du jeûne est annoncée, chaque soir, par un appel à la prière. Cette pratique n'étant pas réalisable en France, les

33. E. Cronier, "Feeding Muslim troops during the First World War ", op. cit.

34. La presse s'en fait même l'écho comme en témoigne cet extrait d'un article intitulé « Heureux fils de Mahomet! » paru dans L'Éclair, quotidien du Midi, le dimanche 25 juillet 1915, en période de ramadan, qui cite la dépêche du ministre de la Guerre : "Il est de coutume de laisser un peu plus de liberté aux militaires musulmans à l'occasion de ces fêtes et d'améliorer, dans la mesure du possible, le repas des soirées qui les précède. Il conviendra de tenir compte de cette coutume [...] Mais quelle chance d'être musulman! Supposez que M. Millerand donne des ordres pour que les soldats catholiques puissent faire le Carême et accomplir rigoureusement, à Pâques, leurs devoirs religieux! Quelle tempête, mes amis, dans les journaux de la "libre-pensée"! Et quelle interpellation à la Chambre ! [...] Pourquoi cette différence ? Je m’en tiens à l'explication que j'ai déjà hasardée : nos gouvernants, tout en étant, comme on sait, attachés à une politique "exclusivement laïque et anticléricale", doivent guigner le paradis de Mahomet. ", p. 1. 
musulmans seront laissés, dans chaque groupement, libres de fixer le moment où ils croiront devoir prendre leur premier repas ${ }^{35}$.

S'agit-il pour autant d'une reconnaissance de la nécessité pour l'armée d'accompagner ses soldats dans leur spiritualité, au sens où l'on pourrait l'entendre aujourd'hui ? Il faut se garder de tout anachronisme. Richard S. Fogarty montre bien que les facilités accordées aux soldats musulmans visent en réalité à éviter toute révolte dans les colonies (qu'en serait-il de l'autorité française si les peuples colonisés venaient à apprendre que leurs coreligionnaires sont mal traités ?) et toute mutinerie au sein des unités de soldats musulmans. La Sublime Porte, alliée de l'Empire allemand, n'a-telle pas lancé un appel à la guerre sainte des musulmans contre les ennemis des puissances germaniques ? En réaction, en juillet $1915^{36}$, les autorités françaises obtiennent du mokaddem (chef) de la zaoüa (confrérie musulmane) de Boghari, en Algérie, une fatwa exhortant les musulmans combattants sous le drapeau français à rester loyaux à la France. "Les autoriser à observer le jeûne du ramadan durant la journée, leur servir leur repas après le coucher du soleil et les informer des fêtes religieuses du mois sacré, cela peut être considéré comme de la "bonne politique". De cette façon, les soldats pouvaient voir des preuves concrètes des précautions prises par l'armée pour respecter leur religion et leurs usages ", conclut Richard S. Fogarty.

\section{Aujourd'hui : entre affirmation identitaire et nécessité de service}

Se dessine l'impression d'un recul entre la Première Guerre mondiale et aujourd'hui, en ce sens qu'il y aurait un problème là où il n'y en avait pas. En réalité, il n'y a plus de régiment d'Afrique ou des colonies et les musulmans sont fondus dans les effectifs (comme les juifs avant eux), tandis que les besoins spécifiques des soldats de confession musulmane sont mieux pris en compte depuis 2005 avec l'arrivée des premières barquettes halal. Auparavant, un menu sans porc "suffisait » et la République appliquait une sorte de repas laïc de substitution, comme dans les écoles publiques : pas de porc mais pas halal.

35. Cité par Richard S. Fogarty, Race \& War in France, Colonial Subjects in The French Army (1914-1918), Baltimore, The Johns Hopkins University Press, 2008, p. 169-201.

36. Voir Service historique de la Défense, SHD GR, 16 N 195. 
Certes, beaucoup de musulmans (et de juifs) s'en contentaient. Ceci a conduit Nathalie Kosciusko-Morizet à dire sur Europe 1, en réaction à une déclaration de Nicolas Sarkozy sur les menus de substitution à l'école ( « double ration de frites » à la cantine proposée par l'ancien chef de l'État à tout enfant qui ne mangerait pas de porc) :

Quand j'étais à l'armée, je me souviens que les pâtés proposés dans les rations de guerre n'étaient pas terribles. Les adjudants s'arrachaient les cheveux parce que la moitié de la promotion se déclarait juive ou musulmane pour éviter le pâté et obtenir des boîtes de sardines! C'est bien la preuve que les menus de substitution existaient déjà et cela ne posait aucun problème ${ }^{37}$.

C'est la demande de menus confessionnels, avec de la viande halal ou casher qui est nouvelle. La sociologue Catherine Wihtol de Wenden analyse très bien le problème ${ }^{38}$. Elle s'enquiert de la situation après plusieurs enquêtes, en 1990 et en 2005, et une directive de Pierre Joxe en 1992 sur le respect des interdits alimentaires :

Originaires du Maghreb dans leur majorité, ces Français de culture musulmane se déclarent plus rarement athées que leurs homologues chrétiens ou juifs car l'apostasie (l'abandon public et volontaire de sa religion) est considérée dans l'islam comme l'une des fautes les plus graves. [...] Certains se disent pratiquants parce qu'ils font le ramadan et ne mangent pas de porc, d'autres refusent de se qualifier comme tels parce qu'il leur est impossible à l'armée de ne manger que de la viande halal, ou parce qu'ils ne réussissent pas à faire les cinq prières par jour : «Je suis plus ou moins pratiquant. C'est vrai que chez moi, je fais ma prière, tout ce qui a à faire. Je fais ramadan bien sûr. Je respecte, c'est-à-dire, je ne mange pas de viande ici (sur la base), je ne bois pas d'alcool. Vous êtes pratiquant? - Je ne dirai pas oui parce que si je disais oui, il faudrait que je fasse les cinq prières par jour. Le jeûne un mois complet je fais. Mais autrement, c'est sans plus. Pour l'alimentation, je ne mange pas de porc, mais autrement, c'est vrai que j'ai fait un compromis par rapport à la viande. ${ }^{39}$

37. Nathalie Kosciusko-Morizet, interview, Europe 1, 11 novembre 2016.

38. Catherine Wintol de Wenden, "Lislam dans l'armée ", Cahiers de la Méditerranée, n 76, 2008, p. 65-88.

39. Ibid., p. 67. 
Dans la définition personnelle du "bon musulman " qu'élabore chaque soldat musulman, s'observe comme une gradation du plus au moins important, inversement proportionnelle aux aspects pratiques. Ce qui paraît le plus important est le plus facile à observer dans le quotidien d'une vie en collectivité : accomplir les cinq prières quotidiennes, ne pas boire d'alcool et ne pas manger de porc sont, en OPEX, des préceptes plus aisés à suivre que de respecter le ramadan et de manger strictement halal. On comprend alors que l'appartenance à l'islam se manifeste à l'extérieur par le refus de manger du porc et de boire de l'alcool.

Catherine Wihtol de Wenden décrit quatre types de comportements parmi les soldats de culture musulmane ${ }^{40}$. Â côté de ceux qui mangent de tout, y compris la viande de porc, se trouve le groupe des non-pratiquants (la grande majorité) qui ne consomment pas la viande de porc et se contentent de cela. Le troisième groupe est celui des soldats qui ne mangent pas de porc et qui souhaitent, pour toutes les autres viandes, ne manger que de la viande halal. Ces soldats opèrent des compromis en raison des nécessités de service. Le quatrième groupe, celui des intransigeants, refuse d'ingérer de la viande non halal. Pour ces soldats, la vie quotidienne s'avère compliquée. C'est là que le rôle du commandement et de l'aumônier est important, avec encore beaucoup de différences selon les endroits. À défaut de trouver des interlocuteurs compréhensifs, ces soldats préparent eux-mêmes leur cuisine, quand ils le peuvent, ou se déclarent végétariens.

On voit qu'on est, au moins sur le plan de l'alimentation, encore loin du souhait d'Abdelkader Arbi, aumônier en chef du culte musulman dans les armées, de "banaliser le fait religieux musulman dans les armées ${ }^{41}$ ". Arrivé d'Algérie en France en 1962, fils d'un sous-officier de carrière et petit-fils d'un poilu mort durant la Première Guerre mondiale, Abdelkader Arbi dirigeait, au moment de sa nomination en 2005, la commission de l'abattage rituel au sein du Conseil français du culte musulman (CFCM) nouvellement créé. Il s'est tout de suite attelé au dossier urgent des repas confessionnels. Cinq ans plus tard étaient fournies les premières boîtes de ration halal.

\section{Ibid.}

41. Philippe Escande, "Abdelkader Arbi : "Je veux banaliser le fait religieux musulman dans les armées" ", Le Monde, 2 juin 2018. 


\section{Où sont aujourd'hui fabriquées les rations des soldats français?}

\section{Étude de marché}

L'organisme qui s'occupe de la logistique alimentaire est le CESCOF (Centre d'expertise du soutien du combattant et des forces), situé à Rambouillet (Yvelines). Il conçoit les rations, commande les produits et les fait livrer à l'ÉLOCA (Établissement logistique du commissariat des Armées), situé aux Ponts-de-Cé près d'Angers (Maine-et-Loire), qui les analyse ${ }^{42}$, les conditionne dans les boîtes de ration, les stocke et les expédie.

Le CESCOF établit les menus et passe un appel d'offres européen. Le dernier a été publié au Journal officiel le 10 juillet 2015 et a été attribué, pour les barquettes conventionnelles, le 11 janvier 2016 et, pour le halal, le 28 mars 2017. Les rations casher ne suivent pas le même circuit en raison des faibles volumes concernés. Le CESCOF n'est pas concerné et c'est l'Économat des Armées (EDA) qui peut ponctuellement passer des commandes, hors marché public. Intéressée par ce marché, la société Bocage Restauration possède une section "plats confessionnels". Elle compte parmi les fournisseurs de l'armée française et indique sur son site internet fournir des menus casher ${ }^{43}$. L'argument avancé par les acteurs qui ont bien voulu nous répondre est celui des trop faibles débits et du refus de certains responsables de commander des boîtes dont les dates de péremption seraient vite dépassées. Pourtant, les rations casher circulent puisque des soldats musulmans relatent que, avant que les rations halal n'existent à partir de 2010, ils demandaient des boîtes casher. Mieux, l'aumônier israélite Moïse Taïeb nous indique la production, il y a une dizaine d'années, de rations halal-casher valables pour les fidèles des deux religions. Cela n'a pas continué, notamment parce que les rations halal/casher ne correspondaient pas totalement au référentiel halal établit en interne par le CESCOF. En effet, il faut toujours garder à l'esprit que si le casher est bien défini ${ }^{44}$, notamment sous l'autorité du Consistoire de Paris, le cahier

42. Le laboratoire accrédité COFRAC vérifie notamment la solidité et l'intégrité des boîtes.

43. http://www.bocagerestauration.fr/domaines-de-competence/plats-confessionnels-halal-kasher/ 44. Le casher est aussi beaucoup plus complexe : le halal n'exige que l'abattage rituel de la viande, l'absence de porc et d'alcool quand, en plus de ces exigences (l'alcool est autorisé mais il doit être 
des charges du halal est beaucoup plus flou et les autorités certificatrices nombreuses.

En 1989, il n'y avait pas à proprement parler de ration halal, mais seulement des rations "sans porc" (Tableau 1 en annexe). Dans ces dernières, les viandes de bœuf et de volaille n'étaient pas issues d'animaux abattus rituellement. Les rations halal proprement dites voient le jour en 2010, soit cinq ans après la création de l'aumônerie musulmane ${ }^{45}$. Aujourd'hui, les menus sans porc existent toujours, à côté des menus halal (Tableau 2 en annexe).

Les quantités commandées en 2016-2017 demeurent fixées dans une fourchette : au minimum 60000 et au maximum 300000 barquettes pour chaque menu halal, 260000 à 650000 pour les menus non-confessionnels, incluant les menus sans-porc et végétariens. Soit un ratio de 1 à 4,3 pour la quantité minimale et de 1 à 2,2 (moyenne 1 pour 3,25) entre les quantités de barquettes halal et les autres. Pour un marché qui n'existait pas avant 2005, la proportion a de quoi étonner. Toutefois, ces données restent théoriques car fondées sur des fourchettes ${ }^{46}$. L'ordre de grandeur est plutôt de 50000 à 80000 barquettes halal par an pour quelque 4 millions de barquettes conventionnelles. Dans chaque boîte individuelle de ration, le militaire trouve le nécessaire pour une journée : la boîte halal contient une barquette de plat cuisiné à base de viande halal et une autre contenant un plat à base de poisson et donc halal par absence de viande (et d'alcool et gélatine de porc). Les besoins pour le casher sont bien moindres et ne justifient en tout cas pas une gestion coordonnée par le $\mathrm{CESCOF}^{47}$. La différence entre les deux évaluations réside sans doute dans le fait que ce marché, aujourd'hui bien maîtrisé, a connu des tâtonnements en raison de son caractère récent et parfois polémique. Il faut aussi rappeler que l'immense majorité des soldats de confession musulmane se contente des menus sans porc. Sur les 17 menus actuels (rations de combat individuelles

casher), la kashrout impose la stricte séparation du lait et de la viande, l'absence de produits issus d'insectes (cochenille) et que tous les instruments de cuisson soient cashérisés.

45. Dès 2005, des plats halal ont été achetés par le ministère de la Défense. Toutefois, le premier appel d'offres pour des conserves individuelles réchauffables de plats cuisinés halal est lancé en 2009 et notifié en 2010 .

46. L'administration s'engage à acheter la quantité minimum et le fournisseur déclare être en mesure de produire la quantité maximum.

47. Entretien avec Romain Pavoni, ingénieur agro-alimentaire dans les vivres opérationnels au CESCOF. 
réchauffables), 7 ne comprennent pas de porc et 3 sont « halal ». En 2014, selon le ministère de la Défense nationale, $6 \%$ des rations de combat étaient « halal».

Les marchés sont passés pour trois ans pour les menus conventionnels et quatre ans pour le halal. La différence vient de l'allongement des délais pour la passation d'appel d'offres pour les produits halal. L'analyse des derniers marchés conclus (uniquement pour les plats, en barquettes de $300 \mathrm{~g}$, réchauffables ou froids) montre le nombre assez faible d'entreprises agroalimentaires qui répondent à l'appel d'offres et le monopole des sociétés françaises. En moyenne, seules trois sociétés répondent, parfois moins, dans certains cas un peu plus, cela dépend du plat : deux seulement pour les crevettes poulet à l'asiatique mais cinq pour la salade mexicaine (froide) ou le bœuf-carottes. Pourquoi les industriels étrangers se mettent-ils très rarement sur les rangs, alors que les marchés, du fait de leur gros volume (deux millions de rations par an), sont nécessairement européens ? La réponse est notamment à rechercher dans une particularité technique ${ }^{48}$ : le marché est toujours passé pour des boîtes métalliques (le format appelé dans le jargon " large hansa 300 g. »). Or, le plat cuisiné appertisé en boîte métallique s'avère un savoir-faire français, tandis que nos voisins européens privilégient soit d'autres matières comme la barquette en plastique, soit d'autres formats (boîte ronde et haute au lieu de plate et rectangulaire), soit encore d'autres méthodes de conservation comme la lyophilisation ${ }^{49}$.

Les critères de choix affichés sont en premier la qualité (pondération $70 \%$ ) et en deuxième le prix $(30 \%)$. L'armée communique sur le fait que la sélection des conserves des boîtes de ration est soumise à un jury de soldats "goûteurs " ${ }^{50}$. C'est sans doute la raison pour laquelle les rations françaises se révèlent si prisées lors des opérations extérieures (OPEX). La valeur d'échange s'établit souvent autour de cinq MRE (Meal Ready-to-Eat) américaine pour une ration française.

48. Cependant, pas exclusivement : des cahiers des charges très pointus en termes de qualité nutritionnelle mais également de qualité gustative des recettes, respectueuses des traditions culinaires françaises, sont un frein évident pour une firme étrangère qui souhaiterait concourir.

49. L'armée française passe aussi commande de repas lyophilisés en poche mais pour des besoins spécifiques et en moindre proportion (12 000 à 36000 sachets pour trois ans). Exemple : BOAMP $\mathrm{n}^{\circ} 13-103260$ (13 juin 2013).

50. "Rations militaires : un concentré de gastronomie ", https://www.defense.gouv.fr/english/ node_64/actu-terre/archives/rations-militaires-un-concentre-de-gastronomie, 5 octobre 2012. 
Les prix hors taxes à l'unité varient de 1,12 euro pour la barquette de Rougail de saucisses (1,20 pour les saucisses de Strasbourg pâtes) à 2,25 pour le navarin d'agneau. Les trois barquettes halal s'avèrent globalement plus coûteuses, à 2,64 (impossible de différencier le tajine d'agneau, les lasagnes pur bœuf ou le chili con carne car le marché est passé globalement à la même société, sans fractionnement par lot. Il s'avère donc impossible de comparer les coûts menu pour menu, par exemple tajine d'agneau halal par rapport à navarin d'agneau conventionnel, ou lasagnes contre lasagnes).

\section{La Bretagne se taille la part du lion}

L'entreprise Larzul ${ }^{51}$, établie dans le pays Bigouden (Finistère), a remporté en 2017 le troisième appel d'offres émis par le CESCOF concernant la fourniture de produits halal pour des soldats français ${ }^{52}$. C'est la première fois qu'un industriel français remporte le marché car les deux premiers marchés avaient été obtenus par la firme italienne Projetto 200053. À l'époque, la société française Bocage Restauration avait candidaté, sans succès. Faut-il voir dans ce succès initial italien la marque de l'avance de l'Italie dans la définition du halal ${ }^{54}$ ? Larzul doit ainsi fournir à l'ÉLOCA des plats cuisinés appertisés halal en barquettes de $300 \mathrm{~g}$. Il est étonnant que cette entreprise qui se targue sur son site internet ${ }^{55}$ d'être spécialisée, outre les pâtés et les soupes, dans les abats cuisinés qui ne sont pas à proprement parler les morceaux prisés par les musulmans (encore moins d'ailleurs des juifs), ait été sélectionnée. Ceci montre bien que la question du goût et de la qualité passe avant la question de la religion. Contrairement à ce qu’il se passe dans l'armée américaine (voir plus loin), les entreprises qui fabriquent les barquettes halal sont généralistes. La plupart ne mettent pas en avant leur rayon halal dans leur communication vers le grand public. La preuve, parmi les sociétés qui fournissent l'armée française, une seule se présente comme spécialisée dans les menus confessionnels halal et casher :

51. L'entreprise Larzul est issue d'une conserverie familiale de viande, créée en 1906 à PlounéourLanvern (Finistère). Dans les années 1960, la troisième génération lance les plats cuisinés. Pâtés, abats cuisinés et bisques de homard font partie de ses spécialités. http://www.groupe-larzul.com 52. Un premier appel d'offre lancé en 2014 avait été remporté par la société italienne mais cette dernière l'a résilié l'année suivante.

53. Progetto 2000 est implantée à Oristano en Sardaigne.

54. Création d'un label «Halal Italia » en juin 2010. En France, le Conseil français du culte musulman (CFCM), créé en 2003, n'est pas parvenu à unifier le marché du halal.

55. http://www.groupe-larzul.com/ 
Bocage Restauration, sise à Loudun dans la Vienne ${ }^{56}$. Elle a remporté le marché pour le porc créole riz ananas et le petit salé lentilles du Puy mais pas pour le halal.

Pour les barquettes conventionnelles, c'est la bretonne CGPA (Compagnie générale de produits alimentaires) Peny ${ }^{57}$ qui écrase le marché. Ce n'est pas étonnant car la CGPA est numéro un des marques distributeurs ${ }^{58}$, notamment grâce à la barquette micro-ondable pour laquelle l'entreprise a été l'une des premières à se positionner au plan national. À elles seules, ces deux entreprises bretonnes Larzul et CGPA-Peny ont remporté 23 lots sur 33 , soit $70 \%$ du marché : plus de 10,2 sur un total de 14,4 M $€$. Sur ce total, le halal représente $2,3 \mathrm{M} €$.

D'aucuns remarquent malicieusement que le ministre de la Défense nationale de l'époque, Jean-Yves le Drian, est breton. Plus prosaïquement, la Bretagne est la première région pour l'agroalimentaire en France et bénéficie du savoir-faire de ses anciennes conserveries et de la proximité géographique de la viande de porc et des légumes. Fort logiquement également, CGPA-Peny et Larzul se sont vus attribuer la fabrication de tous les plats à base de poisson (par ordre décroissant de fréquence : le thon, le saumon et les crevettes).

Dans les choix de Toupnot et de Raynal et Roquelaure, il est permis de voir l'expression de la recherche de la qualité. $\mathrm{R} \& \mathrm{R}$ ( « Je me régale c'est la faute à Raynal, j'en veux encore c'est la faute à Roquelaure ») est considéré comme le nec plus ultra du secteur du plat cuisiné en conserve. La société a été fondée en 1876 par Théophile Raynal, gérant du buffet de la gare de Capdenac ${ }^{59}$, et Ernest Roquelaure, son cuisinier, qui eurent l'idée de conditionner en boîtes de conserve des plats du terroir français. Il est

56. Bocage Restauration est une PME implantée dans le Nord de la Vienne, au cœur du Poitou, de l'Anjou et de la Touraine. Fondée en 1986, la société a été redynamisée par un rachat en novembre 2010. La société est spécialisée dans la fabrication de plats cuisinés appertisés. Elle répond à des marchés spécifiques tels que la diététique, les marchés publics et les plats confessionnels (halal et casher), ainsi que la sous-traitance pour de grands industriels.

57. Sise à Saint-Thurien (Finistère) : 345 employés et 80 millions d'euros de chiffre d'affaires en 2015 .

58. Elle appartient au groupe Cecab depuis 1991. Le groupe Cecab s'appelle Groupe d'Aucy depuis 2015.

59. Le siège de la société $R \& R$ se trouve à Capdenac-Gare, dans l'Aveyron, ville dans laquelle l'entreprise a démarré. Elle compte 570 salariés répartis sur trois sites, localisés respectivement à Capdenac-Gare, Sainte-Livrade-sur-Lot (Lot-et-Garonne) et Camaret-sur-Aigues (Vaucluse). 
étonnant qu'elle n'ait pas gagné à propos du petit salé aux lentilles car la cuisson et la conservation des lentilles demeurent l'une de ses spécialités mais il est moins étonnant qu'elle se soit imposée pour les lasagnes car la société possède une branche orientée vers les spécialités italiennes, à savoir la marque Zapetti. Lancée en 2005 , cette dernière englobe les produits de la marque Buitoni rachetée à Nestlé en 2007 et ceux de Maggi saveurs du monde.

Ces éléments expliquent cette concentration géographique actuelle (Fig. 2).

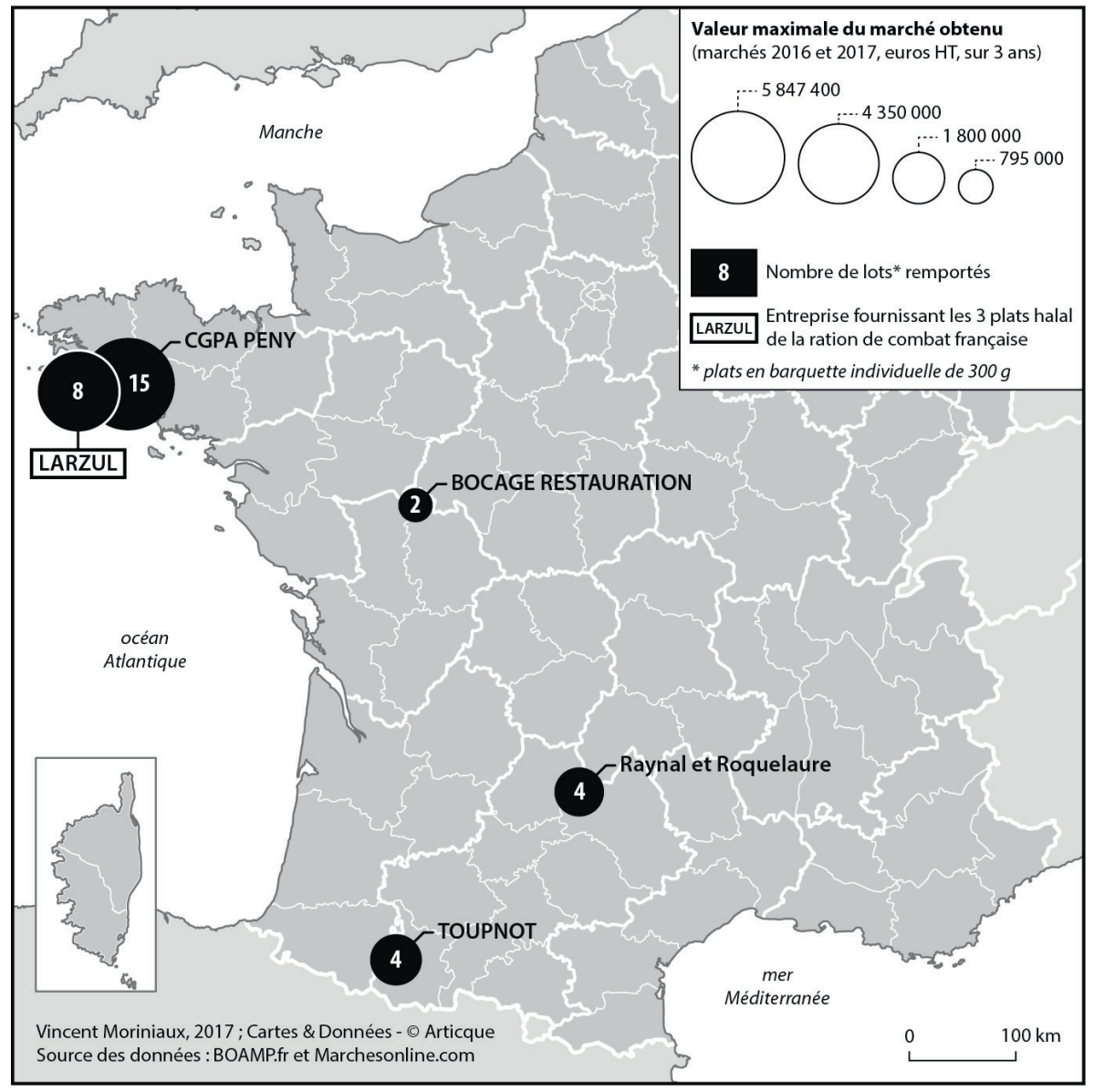

Fig. 2. - Les entreprises fournissant l'armée française en plats cuisinés appertisés, marchés 2016-2017

Source des données : Bulletin officiel des annonces des marchés publics (BOAMP), avis $\mathrm{n}^{\circ}$ 16-34661/2016. 


\section{Que se passe-t-il dans l'armée des États-Unis ?}

Dans l'armée américaine, comme le prouvent les documents officiels (Fig. 3), les menus confessionnels, halal et casher, sont clairement affichés et en nombre égal depuis le milieu des années $1990^{60}$, avec les troupes des États-Unis engagées au Moyen-Orient.

\section{Fig. 3. - Les menus confessionnels dans l'armée des États-Unis d'Amérique}

\begin{tabular}{|ll|}
\hline \multicolumn{1}{|c|}{ Kosher } & \multicolumn{1}{c|}{ Halal } \\
\hline Tuscany Beef w/Cannellini Beans & Lamb \& Vegetable Barley Stew \\
\hline $\begin{array}{l}\text { Hickory Smoked Beef in Brown Rice } \\
\text { with Lentils \& Vegetables }\end{array}$ & Vegetable \& Lamb Jalfrazi \\
\hline Jalapeno Curry Beef w/Potatoes & Beef Roast \& Vegetables \\
\hline Chicken \& Rice & Beef Stew \\
\hline $\begin{array}{l}\text { Spicy Southwest Chicken in Rice w/ } \\
\text { Vegetables }\end{array}$ & Lamb \& Lentil Stew \\
\hline Pasta Marinara & Lentils Dal Marsala \\
\hline Chicken Noodle & SAAG Chole w/Lamb \\
\hline Spicy Vegetarian Chili & Chicken \& Vegetables \\
\hline Chicken Royale w/Brown Rice & Chicken Pesto \\
\hline $\begin{array}{l}\text { Classic Chicken Curry w/Basmati Rice, } \\
\text { Lentils \& Vegetables }\end{array}$ & Chicken Stew \\
\hline New Orleans Gumbo w/Chicken & Penne Pasta \\
\hline $\begin{array}{l}\text { Smoked Beef Paprikash Noodles \& } \\
\text { Vegetables }\end{array}$ & Rattouille \\
\hline
\end{tabular}

Source : Defense Logistics Agency, DLA Troop Support Subsistence, http://www.dla.mil/TroopSupport/Subsistence/Operational-rations/relkoshhal/, avec l'aimable autorisation de la DLA.

Aux États-Unis, l'armée se tourne vers des sociétés spécialisées : une pour le casher, My Own Meals, et une pour le halal, J\&M, qui sont en réalité une même entreprise, fondée en 1991 et située à Deerfield dans l'Illinois. J\&M développe sa propre certification halal et se paie même le luxe de diffuser

60. Military buys special meals for Jewish and Muslim troops, Russell Working, http://www. JewishWorldReview.com, consulté en octobre 2017. 
des normes industrielles pour la fabrication halal ${ }^{61}$, normes qui manquent dans beaucoup de pays, même musulmans, et qui assurent au producteur américain une position dominante.

En France, au lieu de se tourner vers une seule société dont la spécialité serait de fabriquer des plats confessionnels, la qualité et l'origine demeurent privilégiées. Cela pourrait s'appeler l'obsession française du terroir. Ainsi est-il demandé à une entreprise de l'Aveyron, installée depuis 1876 à Capdenac, de préparer un cassoulet ou un plat de saucisses-lentilles. Toutefois, il convient de ne pas oublier qu'une boîte de ration française s'échange contre plusieurs boîtes américaines.

La différence tient également à la conception française de la laïcité, très différente de celle des Américains. L'armée française privilégie l'idée d'une force laïque, où tout le monde est prêt à se fondre dans le même moule pour sauver la patrie, tandis que ne s'exprime aucun communautarisme.

Enfin, la France reste traumatisée par son passé colonial et l'armée évite de communiquer sur des questions qu'elle juge sensibles. En revanche, l'armée américaine joue la transparence car elle a bien compris qu'en distribuant ses rations halal à ses soldats comme aux populations civiles sur le théâtre des opérations, très souvent musulmanes, elle améliore son image comme le suggèrent des photographies du site de J\&M (http:/www.halalcertified.com/).

\section{Conclusion}

La question des rations confessionnelles ne s'arrête sans doute pas là, d'une part à cause de l'évolution technique qui industrialise de plus en plus les rations et en raison des nouveaux défis posés sur le plan religieux. On connaissait déjà le Vinogel (vin concentré et gélifié) dans les boîtes de ration. Sous l'influence de l'armée américaine, la France réfléchit, elle aussi, à des rations sans boîte de conserve, moins lourdes à transporter. Cela écornerait la gastronomie mais que dire du caractère halal ou casher d'un aliment en poudre? D'autre part, diverses postures éthiques prennent peu à peu de l'importance et pourraient ne pas tarder à être revendiquées

61. Halal Industrial Production Standards, janvier 2000 (4 éd.) : http://www.halalcertified.com/ wp-content/uploads/2016/10/Halal-Production-Standards.pdf, consulté le 12 mars 2019. 
d'être traitées à égalité avec les religions établies. Ainsi, en Suisse, en 2016, un soldat vegan, après avoir d'abord été jugé inapte au service, n’a-t-il pas obtenu sa réintégration, à charge pour l'armée de lui fournir un équipement et une alimentation exempts de tout produit animal ${ }^{62}$ ? S'agit-il d'un droit d'objection de conscience d'un nouveau genre ? Il est d'ailleurs intéressant de noter qu'Israël, qui compte aujourd'hui parmi la plus grande communauté de vegans au monde, a été pionnier en la matière et met à la disposition de ses soldats des repas de substitutions sans viande, ni lait, ni œuf, ni poisson.

\section{Annexes}

Comparaison des plats de la ration de combat individuelle réchauffable (RCIR) en 1989 et en 2017

\section{Tableau 1 - Menus de la RCIR en 1989}

\begin{tabular}{|l|l|l|} 
No du menu & \multicolumn{1}{|c}{ Hors d'œuvre } & \multicolumn{1}{c}{ Plats cuisinés } \\
\hline 1 & Volaille en gelée & Bœuf en salade Thon pommes de terre \\
\hline 3 & Rillettes de saumon & Saumon - Riz - Légumes Hachis parmentier \\
\hline 4 & Rillettes de maquereau & Bœuf légumes Poulet riz à l'indienne \\
\hline 5 & Bœuf assaisonné & Sauté de lapin Chili con carne \\
\hline 6 & Thon catalane & Paëlla Veau marengo \\
\hline 7 & Médaillons de sardines & $\begin{array}{l}\text { Agneau aux flageolets Volailles légumes printa- } \\
\text { niers }\end{array}$ \\
\hline 8 & Maquereau à l'escabèche & Navarin d'agneau Poulet riz ratatouille \\
\hline 9 & Mousse de canard & Thon en salade Porc aux lentilles \\
\hline 10 & Pâté de foie pur porc & Cassoulet Cannelloni \\
\hline 11 & Rillettes pur porc & Porc aux légumes Bouf carottes \\
\hline 12 & Terrine forestière & Porc pommes de terre Bouf bourguignon \\
\hline 13 & Pâté de jambon & Porc en salade Risotto aux fruits de mer \\
\hline 14 & Pâté de campagne & Volaille à la parisienne Gratin dauphinois \\
\hline & Rillettes de thon & Saucisses lentilles Moussaka \\
\hline
\end{tabular}

Les menus numérotés de 1 à 7 sont sans porc, ceux numérotés de 8 à 14 sont avec porc.

62. Loan Ego, «Victoire du vegan jugé “inapte” à l’armée », Paris Match, 19 octobre 2016 : http:// www.parismatch.com/Actu/Environnement/Victoire-du-vegan-juge-inapte-a-l-armee-1099013, consulté le 12 mars 2019. 


\section{Tableau 2 - Plats de la RCIR en 2017}

\begin{tabular}{|c|c|}
\hline Plat & Société \\
\hline Petit salé lentilles du Puy & Bocage Restauration \\
\hline Porc créole riz ananas & Bocage Restauration \\
\hline Rougail de saucisses & CGPA-Peny \\
\hline Saucisse de Strasbourg pâtes & CGPA-Peny \\
\hline Tortellini pur bœuf & CGPA-Peny \\
\hline Salade orientale (froid) & CGPA-Peny \\
\hline Pâtes à la carbonara & CGPA-Peny \\
\hline Cassoulet supérieur au canard & CGPA-Peny \\
\hline Thon pomme de terre & CGPA-Peny \\
\hline Taboulé volaille (froid) & CGPA-Peny \\
\hline Bøuf en salade (froid) & CGPA-Peny \\
\hline Thon en salade (froid) & CGPA-Peny \\
\hline Thon piperade & CGPA-Peny \\
\hline Salade de pâtes au saumon (froid) & CGPA-Peny \\
\hline Saumon riz légumes & CGPA-Peny \\
\hline Joue de porc aux ravioles & CGPA-Peny \\
\hline Paëlla & CGPA-Peny \\
\hline Chili con carne & Larzul \\
\hline Boulettes kebab & Larzul \\
\hline Risotto de porc aux champignons & Larzul \\
\hline Salade italienne (froid) & Larzul \\
\hline Crevettes poulet à l'asiatique & Larzul \\
\hline Lasagnes pur bouf (halal) & Larzul \\
\hline Tajine agneau (halal) & Larzul \\
\hline Chili con carne (halal) & Larzul \\
\hline Lasagnes pur bœuf & Raynal et Roquelaure \\
\hline Couscous poulet & Raynal et Roquelaure \\
\hline Parmentier de canard & Raynal et Roquelaure \\
\hline Veau marengo & Raynal et Roquelaure \\
\hline Potée paysanne & Toupnot \\
\hline Bouf carottes & Toupnot \\
\hline Salade mexicaine (froid) & Toupnot \\
\hline Navarin d'agneau & Toupnot \\
\hline
\end{tabular}


Vincent Moriniaux

\section{L'auteur}

Vincent Moriniaux est maître de conférences de géographie à la Faculté des lettres de Sorbonne-Université. Il y enseigne notamment au sein de la spécialité de master «Alimentation et cultures alimentaires ». Il développe des recherches sur la dimension éthique de l'alimentation dans le cadre de l'UMR $8185 \mathrm{ENeC}$ (Espaces, nature et culture).Contact : vincent.moriniaux@sorbonne-universite.fr 